\title{
Análisis de las políticas públicas y los debates en relación a las salas teatrales de la Ciudad de Buenos Aires durante la pandemia
}

\author{
(4) Lautaro Heger \\ ElTyA - Universidad de Buenos Aires, Argentina \\ hegerlautaro@gmail.com
}

Fecha de recepción: 24/08/2021. Fecha de aceptación: 14/10/2021

\begin{abstract}
Resumen
El presente artículo propone reflexionar sobre las medidas desplegadas por el gobierno argentino y de la Ciudad de Buenos Aires, en el contexto de la pandemia de COVID-19, destinadas a la reactivación de las salas teatrales. Se analizarán las diversas formas de incidencia que tuvieron distintos actores pertenecientes al campo teatral, con especial atención en los productores y empresarios pertenecientes al circuito comercial y el circuito independiente, con el fin de garantizar, entre otras acciones, ayudas como el otorgamiento de subsidios, asistencia al pago de salarios y la posibilidad de apertura y realización de funciones.
\end{abstract}

Palabras clave: arte - trabajo artístico - pandemia - políticas culturales - salas teatrales

\section{Analysis of Public Policies and Discussions Related to Theaters in the City of Buenos Aires During the Pandemic}

\begin{abstract}
This article reflects on the measures implemented by Argentina's national government and by the government of the City of Buenos Aires in the context of the COVID-19 pandemic to promote the reactivation of theaters. We will consider the various forms of incidence that different actors of the theater field -particularly, producers and entrepreneurs of the commercial and independent circuits- had in order to guarantee, among other actions, aid such as the granting of subsidies, assistance with the payment of salaries and the possibility of re-opening.
\end{abstract}

Keywords: art - artistic work - pandemic - cultural policies - theater 


\section{COVID-19 y cultura: ¿de dónde venimos y hacia dónde vamos?}

Durante el 2020, el contexto de pandemia que hoy persiste desestabilizó las distintas esferas sociales. A nivel internacional, la situación dejó en evidencia las enormes desigualdades entre los países. En el caso de Argentina, el panorama socio-económico se vio nuevamente resentido por la inminencia de la COVID-19, las medidas de confinamiento prolongado y la persistencia de las consecuencias de las políticas neoliberales, desplegadas entre el 2015 y 2019 por el gobierno de la alianza política Cambiemos.

Muchos sectores productivos se vieron imposibilitados de continuar con su actividad normal. La cultura en general, y las artes escénicas en particular, se encontraron (y se encuentran) fuertemente impactadas por los factores mencionados anteriormente, tanto en la producción de obras teatrales como en el consumo y asistencia por parte del público. El 20 de marzo de 2020, fecha de inicio del Aislamiento Social, Preventivo y Obligatorio (ASPO) dispuesto por el Poder Ejecutivo Nacional, marcó el cierre de instituciones culturales y la interrupción de actividades artísticas de forma presencial. De esta forma, espacios como cines, teatros, salas de conciertos y otros dedicados a la producción y circulación de bienes culturales iniciaron una paralización de su trabajo cotidiano y la agudización de las dificultades económicas, como consecuencia de la falta de generación de ingresos y la imposibilidad de hacer frente a los gastos de alquiler, mantenimiento, impuestos y servicios que continuaron pese a la ausencia de actividades en esos espacios.

Sin embargo, esto no representó una inercia de los artistas y los trabajadores de la cultura. En lo que se refiere a organizarse laboral y políticamente, se destaca el surgimiento de asociaciones profesionales que agrupan a los artistas de acuerdo a la actividad que llevan a cabo en las distintas disciplinas. Dichos espacios se han ocupado de visibilizar las necesidades y las problemáticas que conciernen a sus representados. También han colaborado con la realización de censos y otros mecanismos para el relevamiento de datos y estadísticas. El objetivo era que las administraciones culturales del Estado pudieran contar con información para llevar adelante las políticas públicas y hacer frente a la imposibilidad de los trabajadores de la cultura de desarrollar su actividad con normalidad. A su vez, se buscaba dar respuestas al sector respecto de ciertas luchas y reivindicaciones que se vienen arrastrando desde hace mucho tiempo.

En el presente trabajo se analizará en detalle la situación de las salas teatrales de la Ciudad de Buenos Aires: cuál era el panorama de las mismas antes de la pandemia, qué medidas se impulsaron desde las administraciones culturales del gobierno nacional y la ya mencionada Ciudad de Buenos Aires y cómo repercutieron dichas políticas públicas en el sector. Mediante el relevamiento bibliográfico, notas periodísticas, artículos de divulgación, datos compilados en informes elaborados por instituciones públicas y organizaciones que nuclean la actividad y la representación de las salas teatrales y los trabajadores de la cultura de acuerdo a su actividad y la realización de entrevistas a directores y dueños de salas, se ofrecerá un panorama integral de las problemáticas circundantes al trabajo y la organización de los teatros, las consecuencias de su cierre durante el inicio y desarrollo de la pandemia y la posterior apertura, a comienzos del 2021, y sus implicancias en términos concretos y simbólicos.

\section{Las salas y circuitos teatrales de la Ciudad de Buenos Aires}

Para iniciar el recorrido por el análisis de las políticas desplegadas hacia las salas teatrales de la Ciudad de Buenos Aires durante la pandemia, resulta necesario definir dos elementos correspondientes al mencionado objeto de estudio. Por un lado, qué son y cómo se configuran las salas teatrales en relación a sus espacios 
y actividades. Por otro lado, de qué se habla cuando se hace mención al circuito alternativo o el circuito comercial y qué rol ocupan las salas en el mismo. Los autores a desarrollar en los párrafos siguientes corresponden a las reflexiones desplegadas por la investigadora Karina Mauro (2018), las cuales ofrecen conceptos y herramientas metodológicas para abordar las especificidades del trabajo artístico y cultural.

En referencia al primer elemento, es posible encontrar diversas definiciones que dan cuenta de algunas características que ilustran no sólo a las salas teatrales como espacios físicos sino, también, en las funciones y los objetivos que cumplen las mismas como agentes del campo cultural (Bourdieu, 2005). En el trabajo elaborado por Alejandro Rozenholc (2015) se destaca que algunas salas del circuito alternativo comenzaron a surgir después de la crisis del 2001, con el reciclaje de grandes casas y otros espacios (en su mayoría, galpones) que, en algunos casos, proponían un nuevo vínculo con el espectador. Es allí donde dicho autor marca una diferencia con las salas teatrales comerciales que, en su mayoría, poseen una distribución conocida como teatro a la italiana. Otro detalle relevante que se agrega a esta caracterización es que las salas autogestionadas, al encontrarse sujetas a la Ley Nacional del Teatro ( $\mathrm{N}^{\circ} 24800$ ), las mismas no deben superar las trescientas localidades. Diferente es el caso de las salas comerciales, donde los espacios superan ampliamente ese número, llegando a superar, en algunos casos, la barrera de las mil quinientas localidades.

En relación a sus funciones, Rozenholc (2015) les otorga un rol primordial como agentes del campo teatral en lo referido a la fase de explotación o exhibición de un espectáculo. Los criterios de programación son variados y pueden estar relacionados con factores estéticos (calidad artística de la propuesta, lugar dentro del campo teatral del director y/o los actores, nacionalidad del autor, etc.), político-burocráticos (diseño y cumplimiento de una programación con el objetivo de obtener determinados subsidios estatales) y económicos. Estos factores se encuentran en los teatros alternativos y explican la diversidad de actividades que acontecen en una sala, además de los espectáculos teatrales, como cursos, talleres, ofertas gastronómicas, entre otras y, también, la dinámica de rotación rápida de las obras para garantizar una mayor afluencia de público y, en consecuencia, mayores ingresos. En el caso del teatro comercial, se destacan los factores económicos y, muchas veces, los factores estéticos se encuentran sujetos a estos.

Otro autor que ofrece características que definen a las salas teatrales es Rubens Bayardo (1997). En su trabajo en el que denomina al teatro alternativo como OffCorrientes, establece una clara oposición en sus lógicas estéticas, económicas e, incluso, edilicias y geográficas con el llamado teatro comercial. Bayardo propone una descripción de las salas teatrales, a partir de quiénes son los encargados de administrarlas, cómo y cuáles son las actividades realizadas y con qué dinámicas se aseguran estos espacios sus ingresos y recursos económicos.

El autor plantea como una característica relevante de las salas de teatro del circuito alternativo el hecho de que, en su mayoría, las mismas están administradas por artistas, diferenciándose de los grandes empresarios de paredes que predominan en el circuito comercial. Esto explica, en algunos casos, la existencia de espacios que, además de contar con lugares diseñados y utilizados para la exhibición de obras teatrales, también son utilizados por los artistas que los coordinan para instalar sus estudios o instancias de formación con talleres de distintas disciplinas teatrales (actuación, dramaturgia, dirección, etc.). A su vez, funciona como justificación de la otra denominación con la que Bayardo alude al teatro independiente, bajo el nombre de teatro de actores. 
La otra cuestión en la que el autor se detiene es en el mecanismo por el cual las salas teatrales generan sus ingresos. La principal fuente de dinero de los espacios alternativos es la que deriva del porcentaje de lo recaudado por cada función en la boletería, el cual, en algunos casos, viene acompañado de un monto mínimo a cubrir, conocido como seguro de sala. A su vez, los recursos económicos pueden provenir del alquiler de las salas para la realización de ensayos, los aranceles de los talleres impartidos y, si la sala cuenta con bar, también se suman los consumos de los alimentos y bebidas. En los teatros comerciales, además de la recaudación por la venta de entradas, también obtienen ingresos por el alquiler de las salas para eventos corporativos.

Lo siguiente es caracterizar al circuito comercial y al circuito alternativo. Los mismos han sido mencionados al inicio de este apartado y, a partir de la caracterización sobre el funcionamiento y el rol de las salas teatrales, realizada anteriormente, han surgido algunos rasgos en la delimitación de los componentes de cada sistema de producción y circuito de exhibición.

En el circuito comercial, la característica principal es que se trata de producciones encaradas por productores individuales o empresas dedicadas, integralmente, a la realización de espectáculos. Resulta interesante que, hoy en día, es frecuente la asociación de estos empresarios con otras compañías pertenecientes a rubros absolutamente ajenos al mundo del espectáculo (en su mayoría, empresas aseguradoras y/o del rubro automotriz). Dichas compañías no sólo llevan adelante su inversión como aportantes de recursos o en calidad de sponsors en las obras teatrales que se realizan sino, también, en el financiamiento de las salas y espacios teatrales que albergan dichas obras.

El objetivo que persigue el sistema de producción y el circuito comercial es asegurar la mayor rentabilidad económica posible. Esto explica la utilización de diversas estrategias con el objetivo de atraer a la mayor cantidad de público, entre las que se destacan la contratación de actores, actrices y/o figuras con un alto nivel de reconocimiento por sus trabajos en otros medios como, por ejemplo, la televisión y las redes sociales y la accesibilidad a los grandes medios de comunicación para la difusión de las obras. En lo que refiere a las salas teatrales pertenecientes a este circuito, además de los elementos ya mencionados, se destaca la gran cantidad de personal fijo, como boleteros, acomodadores, operadores técnicos y demás trabajadores, lo que implica una importante inversión en salarios, que se suman a los correspondientes a los equipos artísticos de cada espectáculo a realizar.

En relación a los elementos del circuito alternativo o independiente, resulta necesario reponer algunos momentos históricos que marcaron un rumbo en el surgimiento y posterior desarrollo de dicho sistema de producción. La fecha que determina el inicio es el 20 de marzo de 1931. Ese día, Leónidas Barletta da comienzo a la primera organización teatral independiente, con un claro posicionamiento en sus principios éticos, artísticos y culturales que pueden ser resumidos en un carácter cooperativo en la organización del trabajo de los creadores, con total independencia de los empresarios teatrales y, también, libertad en los procesos creativos. Si bien, hoy en día, algunos de los preceptos delimitados por Barletta ya se encuentran superados $y$, en parte, cuestionados, dichas pautas resultaron muy significativas para la creación de muchas agrupaciones que ponderaban el enfoque colectivo para la producción de obras teatrales.

La segunda fecha que resulta imprescindible conocer corresponde a 1968. Ese año, la Asociación Argentina de Actores, frente al predominio considerable de los grupos independientes, diseña un reglamento con el objetivo de ofrecer una primera regulación de la actividad en las cooperativas teatrales que, a partir de la puesta en marcha del mismo, son incorporadas al régimen laboral en consonancia con las leyes 
del trabajo y los convenios colectivos correspondientes, bajo la figura legal de las Sociedades Accidentales de Trabajo. Si bien en la jerga cotidiana se sigue haciendo alusión a estos agrupamientos como cooperativas, técnicamente no ostentan la misma lógica de funcionamiento. Las SAT son modos de organización que reconocen el carácter eventual de la actividad. A su vez, aunque exista la idea de una inversión de capital económico por parte de sus integrantes y, durante un tiempo aunque ya no en vigencia, la figura del productor se contemplaba en cuanto a su rol como aportante de recursos para el montaje del espectáculo, la recuperación de dicha inversión durante el período de exhibición y explotación, en la mayoría de los casos, resulta incierta y, en caso de que la misma se concreta, siempre implica la resignación del porcentaje de ingresos asignados por quienes integran estas sociedades.

En la década del noventa, con el surgimiento del Instituto Nacional del Teatro en Argentina y el Instituto Proteatro en la Ciudad de Buenos Aires, la esfera estatal realiza un aporte desde el cual se posiciona en su función de apoyo y fomento a la actividad teatral no oficial y no comercial, con líneas de subsidios y financiamiento para grupos teatrales, estables y eventuales, y para salas y espacios culturales del circuito alternativo. La aparición de estas instituciones puso en tensión la categoría de teatro independiente, ya que mediante la obtención de los subsidios se entiende una participación del Estado en la realización de los espectáculos.

A partir de estos acontecimientos concernientes al surgimiento y desarrollo del circuito y sistema de producción del teatro independiente, es posible dar cuenta del mismo como un enfoque en el que se destacan la dimensión colectiva del trabajo y la producción artística, la innovación y la libertad en relación a las propuestas y los procedimientos estéticos. Sin embargo, presenta muchas tensiones en cuanto al reconocimiento laboral y económico se refiere, ya sea por los bajos o nulos ingresos que los artistas y trabajadores de este circuito perciben o por cómo las situaciones de eventualidad y precarización, moneda corriente dentro del teatro alternativo, conllevan a una distorsión de la identidad de los artistas y productores culturales como trabajadores.

\section{Políticas públicas dirigidas a las salas teatrales de la Ciudad de Buenos Aires en pandemia}

Las salas teatrales teatrales se enfrentan, en pleno contexto de pandemia, no sólo al surgimiento de nuevas problemáticas como consecuencia de la paralización total de sus actividades sino, también, a la agudización de otros conflictos que el sector viene arrastrando desde hace mucho tiempo. Una gran parte de los trabajadores de la cultura se han organizado y han relevado datos para contribuir a las administraciones culturales del estado en el diseño e implementación de políticas públicas como en la interpelación hacia los actores políticos para reclamar su intervención y una mayor interiorización en los aspectos concernientes a la dimensión laboral y económica del trabajo artístico y cultural.

Alejandro Grimson (2014) afirma que en el discurso social imperante existe un predominio de la dimensión decorativa de la cultura. A su vez, menciona que esta concepción resulta problemática si se tiene en cuenta el incremento de participación en el Producto Bruto Interno que la cultura ha adquirido en los últimos años y que, por ejemplo, en la Ciudad de Buenos Aires alcanza un $11 \%$. Frente a esta perspectiva, el autor da cuenta del reduccionismo al que, constantemente, deben hacerle frente los artistas, productores y otros trabajadores de la cultura al momento de llevar adelante acciones e iniciativas por el reconocimiento de sus derechos. De esta manera, se explica el escaso rebote mediático que han tenido sus reclamos en el contexto de pandemia, aunque también podría aplicarse a otros contextos ordinarios. 
En el siguiente apartado, se expondrá respecto de las medidas y políticas públicas desplegadas, por las autoridades culturales a nivel nacional y municipal, hacia las salas teatrales de la Ciudad de Buenos Aires en el contexto de pandemia, con especial detalle en los diversos actores que participaron en el diseño y la implementación de las mismas. Resulta interesante destacar el aporte realizado por Alejandra Carpineti (2021), directora del Teatro La Carpintería y vicepresidenta de ARTEI, asociación civil con más de veinte años de trayectoria que nuclea y representa a más de cien salas autogestivas de la Ciudad de Buenos Aires. Carpineti realiza un recorrido por los distintos reclamos que vienen llevando a cabo, a partir de diversas problemáticas como, por ejemplo, las dificultades que comenzaron a presentarse en relación a la habilitación de los espacios culturales, después de la tragedia acontecida en el local bailable República Cromagnon, el 30 de diciembre de 2004. También, menciona algunas de las medidas puestas en funcionamiento, con el fin de mitigar el impacto económico de la pandemia y de las que, a continuación, se hará un repaso y análisis respecto de cada una de ellas.

Entre las medidas de alcance general con impacto en las salas teatrales, se encuentran la suspensión de desalojos, la prórroga de los contratos de locación, el congelamiento de precios de los alquileres y, en caso de contar con la posibilidad de poder afrontar dichos gastos, poder hacerlo a través de transferencias bancarias o depósitos por cajeros automáticos. Dichas facilidades rigieron hasta el 31de marzo de 2021 y fueron contempladas en el Decreto de Necesidad y Urgencia 320/20, en cuyo artículo 9 inciso 3 detalla el alcance del mismo a inmuebles destinados a actividades culturales o comunitarias. Como otra medida a destacar, se encuentra la abstención de cortes de servicios tales como internet, televisión por cable, telefonía fija, gas, electricidad y agua. Además de la suspensión en los cortes de los suministros de dichos servicios, se contemplaron el acceso a diversas facilidades de pago y la solicitud de alta o renovación de la denominada Tarifa Social. En la situación particular de la Ciudad de Buenos Aires, también se incluyó la exención de las cuotas correspondientes a Julio y Agosto del 2020 del impuesto conocido como Alumbrado, Barrido y Limpieza $(\mathrm{ABL})$, establecida por la Ley de Alivio Fiscal ante la Pandemia Coronavirus $\left(\mathrm{N}^{\circ}\right.$ 6315). Lo interesante es que, si bien, se trataron de medidas que buscaron paliar el impacto de las deudas correspondientes a impuestos y servicios, Carpineti no las menciona como iniciativas que hayan representado un alivio. La liquidación de dichos servicios y tributos continuaron y se acumularon por la falta de actividad de las salas teatrales, constituyendo, en muchos casos, el motivo principal de cierre de algunos de esos espacios.

Una de las cuestiones que resultaron polémicas ante la comunidad artística es la falta de medidas de carácter urgente por parte del Gobierno de la Ciudad de Buenos Aires, lo que llevó a muchos trabajadores de la cultura que se desempeñan en el circuito alternativo a impulsar campañas para declarar la emergencia cultural en dicha ciudad. Sin embargo, lo que se destaca por parte de ARTEI es la puesta en marcha de las convocatorias a subsidios para el fomento de la actividad teatral no oficial por parte del Instituto Proteatro, dependiente del programa Impulso Cultural. Dicho instituto ofrece, entre otras líneas de financiamiento, un subsidio destinado a las salas teatrales del circuito alternativo con el objetivo de llevar a cabo arreglos y mejoras edilicias en los espacios, gastos de gestión y programación o adquisición de equipamiento. Entre los requisitos a cumplir por quienes solicitan esta subvención se encuentran la consignación de la sala dentro del registro del Instituto Proteatro, contar con un usuario en el sistema de Tramitación a Distancia (TAD), título de propiedad o contrato de alquiler, comodato o convenio, habilitación de la sala expedida por la Agencia Gubernamental de Control (AGC), antecedentes de actividades y programación de espectáculos desarrollada y a desarrollar en un período no menor a nueve meses con por lo menos tres funciones semanales los 
viernes, sábados y domingos, en horarios centrales; mínimo una obra de autor nacional o extranjero con mínimo de cinco años de residencia ininterrumpida en Argentina, entre otras disposiciones.

Si bien se destaca, por parte de ARTEI, la posibilidad ofrecida por Proteatro de realizar dicha presentación, el inconveniente principal que se presentó durante el 2020 fue que los montos de los subsidios resultaron insuficientes frente a la inactividad de las salas y, a su vez, no hubo contemplación en la urgencia de la necesidad de contar con esa subvención en el menor tiempo posible. A su vez, Carpineti menciona que, año tras año, el presupuesto destinado al Instituto Proteatro expresa una tendencia a la baja. Esa es la mayor preocupación por parte de ARTEI y una de sus principales reivindicaciones, la cual se profundizó durante la pandemia, debido a la necesidad de utilizar las ayudas para mantenimiento general de las salas y, en la medida de lo posible, tratar de llevar a cabo las refacciones correspondientes para impulsar actividades, con la contemplación de estrictos protocolos sanitarios. De la convocatoria 2020 correspondiente a salas teatrales y espacios independientes, las solicitudes aprobadas fueron ciento cincuenta y cuatro.

Además de los subsidios ofrecidos por Proteatro, también se encuentran las líneas de financiamiento ofrecidas por el Fondo Metropolitano de la Cultura, las Artes y las Ciencias. Las mismas estaban orientadas a cubrir el pago de los servicios y gastos de mantenimiento de espacios culturales que, en el caso de las salas teatrales, tenían como monto máximo la suma de \$360.000 en la convocatoria correspondiente al 2020 y, en la convocatoria 2021, la suma máxima a solicitar llegaba a los \$400.000. De la convocatoria correspondiente al 2020, las solicitudes aprobadas para espacios culturales fueron 139 , aunque el porcentaje que corresponde a salas teatrales es minoritario, ya que abarca otros espacios como salas de concierto, galerías de arte, bares notables, entre otros.

Esto, de alguna manera, permite dar cuenta de la prioridad que los artistas y productores que se encargan de administrar y coordinar las salas del circuito alternativo le otorgan a la convocatoria correspondiente al Instituto Proteatro. Esto es debido a que dicha línea de financiamiento no impone un monto mínimo ni máximo a solicitar, por lo menos de forma explícita. A su vez, la presencia de representantes de las salas teatrales en el directorio del instituto, que participan en la evaluación en detalle y la posterior aprobación de los proyectos, representa un voto de confianza ya que la voz de las organizaciones se encuentra presente, con la intención de contemplar a la mayor cantidad de presentaciones, pese al ya mencionado descenso del presupuesto.

Así como se han abordado las medidas encaradas desde el Ministerio de Cultura de la Ciudad de Buenos Aires, es necesario dar cuenta de las políticas públicas desarrolladas por la administración cultural a nivel nacional. A diferencia de lo analizado en relación a la gestión municipal, que se limitó a la apertura de las convocatorias habituales o de carácter ordinario, la gestión a nivel nacional se abocó al desarrollo y la implementación de ayudas económicas de carácter extraordinarias, además de adaptar las convocatorias desarrolladas años anteriores a proyectos escénicos que puedan elaborarse en contextos digitales $\mathrm{y} / \mathrm{o}$ virtuales.

Entre las medidas implementadas por el gobierno nacional que constituyeron una ayuda considerable por parte de las asociaciones y organizaciones que nuclean a las salas teatrales de la Ciudad de Buenos Aires, y el resto de los distritos del país, se encuentra el Plan Podestá. Impulsado por el Instituto Nacional del Teatro como plan de preservación operativa de elencos, salas y teatristas argentinos, lo que Carpineti destaca de este plan es el carácter expeditivo en la evaluación de las solicitudes y la 
rápida respuesta que tuvo, la cual sucedió a una semana de decretado el confinamiento estricto y permitió una primera forma de contención frente al cierre y suspensión intempestiva de actividades culturales.

Otra de las ayudas impulsadas, en este caso directamente implementada por el Ministerio de Cultura de la Nación, es el Fondo Desarrollar. Este fondo, que se amplía a espacios más allá de las salas teatrales, se focaliza en ayudar a la continuidad de la producción, formación, investigación y promoción del arte y la cultura, en cualquiera de sus manifestaciones. Sobre esta convocatoria, ARTEI destaca que muchas de las salas que ellos representan obtuvieron asistencia por parte de este fondo y que, a su vez, aquellas salas asociadas que no fueron seleccionadas estuvieron contempladas en una subvención similar, con el mismo nombre y articulada a través de una negociación realizada entre ARTEI y el Instituto Nacional del Teatro. Cabe destacar que, en esta convocatoria, existían montos máximos de acuerdo con la cantidad de localidades que los espacios seleccionados disponen. Para espacios con hasta cien localidades, el monto máximo a otorgar era de \$150.000. Para espacios con capacidad de entre cien y doscientas personas se otorgaba la suma de hasta \$200.000 como máximo. Por último, los espacios con capacidad de entre doscientas y trscientas personas, el monto máximo a solicitar correspondía a la suma de $\$ 250.000$.

Entre los meses de febrero y marzo del 2021 se sumó el plan denominado Reactivar Escena. El objetivo fue impulsar el retorno a la actividad de salas teatrales y musicales. De allí se desprende la articulación de este programa encarado por el Instituto Nacional del Teatro y el Instituto Nacional de la Música. Si bien, el propósito de esta ayuda estaba encuadrada en el incipiente retorno de las actividades culturales durante el inicio del mencionado año, frente a un contexto epidemiológico que comenzaba a ser favorable a la apertura de algunos sectores productivos, rápidamente y frente a nuevos ascensos en las curvas de contagios y muertes, especialmente a comienzos del mes de abril, los teatros y espacios culturales se vieron forzados a cerrar nuevamente sus puertas hasta que las condiciones sanitarias permitieran una nueva apertura. De ahí surgió la preocupación de las asociaciones de salas tanto frente a aquellos espacios que se presentaron al plan Reactivar Escena en cuanto a la asignación de los montos aprobados. En ese sentido, ARTEI se encuentra todavía trabajando respecto de cómo continuará el desarrollo de este plan.

En relación a las salas del circuito comercial, la preocupación que tuvo más prioridad entre los empresarios fue la de sostener los puestos de trabajo con los que dichos espacios cuentan. Pese al mencionado apoyo de las empresas privadas, en calidad de sponsors, el gobierno nacional desarrolló dos medidas que permitieron la cobertura de salarios de boleteros, acomodadores, operadores técnicos, entre otros trabajadores fijos de los teatros comerciales. La primera fue la denominada Asistencia al Trabajo y la Producción (ATP), que tenía como objetivo el aporte, por parte del gobierno nacional, de hasta el $50 \%$ del total del salario de los trabajadores del sector privado. La segunda fue el Programa de Recuperación Productiva (REPRO), que también consistía en la asistencia al pago de salarios, con la diferencia de estar orientado a las actividades económicas que se encontraban en estado crítico, como el turismo y la cultura.

Dentro de todas estas iniciativas se observa no sólo el carácter urgente en el diseño y la implementación de algunas medidas destinadas a la contención económica del sector de las artes escénicas o tendientes a fomentar su retorno a la actividad. También se da un llamado de atención que Néstor García Canclini (1987) realiza de forma acertada que implica no reducir el trabajo de las políticas culturales a un ámbito exclusivamente gubernamental. Muchas de las medidas analizadas en este apartado corresponden a procesos en los que, constantemente, las distintas asociaciones y organizaciones de 
la actividad escénica han influido y, por momentos, contribuido hacia un enfoque democratizador, pese a las dificultades en cuanto a la falta de datos, estadísticas y, sobre todo, recursos presupuestarios.

\section{Abran los teatros: surgimiento y organización}

Si bien existieron, como se mencionó en el apartado anterior, una serie de medidas desplegadas por las administraciones culturales a nivel nacional y municipal, la respuesta de los artistas y gestores evidenciaba que esas ayudas resultaban insuficientes. Los principales reclamos estaban asociados a los montos y el acceso a las ayudas económicas, o que las mismas no contemplaban el carácter de urgente tan preponderante del contexto.

Durante mediados de 2020 distintas asociaciones, organizaciones y productores del circuito teatral comercial y alternativo llevaron adelante campañas para solicitar la apertura de las salas teatrales. Al mismo tiempo, se comenzó con un trabajo colectivo para el diseño de un protocolo que permitiera el regreso del público y de los elencos. En un principio, la discusión por la confección del protocolo fue cuestionada y eludida por la Asociación Argentina de Actores. Dicha organización manifestó un acatamiento total a las medidas de cuidado y restricción de actividades presenciales, pese a los reclamos esgrimidos por algunos de sus afiliados y otras organizaciones del sector. También se llevaron a cabo algunas movilizaciones por parte de empresarios y artistas, aunque sin mucha repercusión y nivel de convocatoria dentro del propio colectivo artístico.

Durante el mes de noviembre de 2020, los protocolos elaborados por las asociaciones artísticas, supervisadas y aprobadas por la administración estatal, se pusieron en marcha. Sin embargo, dicha implementación sólo pudo ser concretada por aquellas salas del circuito comercial, debido a que las dimensiones de las mismas permitían el aforo del $30 \%$ y, a su vez, podían garantizarse un ingreso mínimo por venta de entradas. Distinta fue la suerte del teatro autogestivo, cuyas limitaciones en cuanto a la cantidad de localidades y la merma de las recaudaciones tuvieron que permanecer cerradas o, en el mejor de los casos, continuar con actividades en formato virtual.

El inicio de 2021, debido a la alentadora situación epidemiológica, otorgó un margen para desarrollar actividades implicadas en la clásica temporada de verano. Esto significó un asentamiento de la actividad teatral comercial en cuanto a la implementación del protocolo y representó un primer intento de apertura para las salas del circuito independiente. Más allá del acompañamiento de las organizaciones del teatro independiente al reclamo de la apertura de los teatros y su participación en la confección del protocolo, el regreso a la actividad, en palabras de Alejandra Carpineti, fue más simbólico que un hecho concreto para el circuito alternativo. Los números aportados por ARTEI indican que sólo el 17\% de las salas autogestivas pudieron retornar a la actividad durante el período de gracia impulsado en los primeros meses de 2021.

A su vez, dentro de estas mismas salas, fueron pocas las que pudieron realizar actividades o funciones de forma virtual. En ese sentido, resulta interesante la apreciación de Carpineti, donde menciona que no existieron muchas ayudas económicas que estuvieran enfocadas en la producción de contenidos teatrales para ser exhibidos vía streaming. Lo más cercano que existió a eso último fue la gestión de permisos de circulación y autorización, por parte del gobierno nacional y de la ciudad, para la realización de actividades culturales al aire libre o la producción de funciones para su exhibición en formato digital. 
En relación al circuito comercial, el nuevo cierre establecido debido a la suma de las estadísticas epidemiológicas, representó cierto malestar por parte de algunos empresarios. Por un lado, argumentaban la imperiosa necesidad de la vuelta al trabajo de aquellos artistas que no cuentan con un ingreso más allá de las funciones que realizan. Por otro lado, si bien obtuvieron ingresos, estos no lograron cubrir los sueldos del personal fijo de las salas, a lo que se sumó la dificultad de hacer frente a las deudas arrastradas del 2020 y acumuladas debido a meses de falta de actividad.

En la actualidad, con el proceso de vacunación en desarrollo, la situación epidemiológica fue permitiendo el aumento del aforo para las salas teatrales. A su vez, la implementación de los protocolos sanitarios funciona como parte del trabajo de concientización de las salas y empresarios teatrales para fomentar la asistencia al teatro.

\section{A modo de conclusión}

Resulta un tanto complejo elaborar sentencias respecto de un fenómeno que, hoy en día, continúa en su desarrollo y, también en sus consecuencias. Sin embargo, como se mencionó al inicio de este trabajo, la pandemia de COVID-19 llegó para modificar las distintas esferas sociales y las actividades humanas. En ese sentido, el trabajo académico no puede (y no debe) ser la excepción.

El contexto actual reveló la importancia de la elaboración de políticas culturales, las cuales resultan imprescindibles no sólo para resolver los problemas que han surgido con la pandemia sino, también, para llevar adelante un diagnóstico claro e integral sobre los conflictos y las luchas históricas con el fin de mejorar las condiciones laborales y de producción en el ámbito del arte y la cultura.

En el conjunto de asociaciones civiles y organizaciones que nuclean la actividad y la representación de salas comerciales y alternativas de la Ciudad de Buenos Aires es posible encontrar agentes del campo cultural que cuentan con una aceitada trayectoria en materia de generación de acciones e iniciativas que lograron la conquista de muchas reivindicaciones como la participación en la reglamentación de la habilitación de las mismas y la discusión, año tras año, de los requerimientos presupuestarios para garantizar una llegada mayoritaria de las líneas de financiamiento ofrecidas por los diversos institutos encargados del fomento de la actividad teatral no comercial y no oficial.

Pese al rol y al lugar que el estado nacional y municipal le brinda a estas asociaciones para la recepción y resolución de sus reclamos, la pandemia ofició como un contexto que evidenció las limitaciones del sector que refiere a las todavía problemáticas concernientes a la precarización predominante del ámbito teatral, en sus distintos sistemas de producción. Sobre estos aspectos, por lo menos continúan las reflexiones para que, en un futuro no tan lejano, se traduzca en acciones y políticas públicas en lo concreto. 


\section{Dibliografía}

》 Alonso, P. (2020). Políticas culturales en la Argentina: un recorrido por sus principales planes (1983 - 2019), Argentina: Cuadernos del INAP.

"Bayardo, R. (1997). El teatro "off corrientes": ¿una alternativa estético-cultural?, Tesis de Doctorado.

» Berstein, B. (2020). “Artes escénicas en tiempos de pandemia: tres oportunidades para repensar más allá de la crisis". Buenos Aires: RGC Ediciones (en línea). Consultado el 8 de junio de 2021. Disponible en https://bit.ly/3504Mpb

» Bonet, L. (2020). "Reflexiones sobre el impacto del COVID-19 en la cultura". Buenos Aires: RGC Ediciones (en línea). Consultado el 2 de junio de 2021. Disponible en https://bit.ly/35obwmV

» Bourdieu, P. (2005). Capital cultural, escuela y espacio social, Buenos Aires: Siglo XXI.

"Cruz, A. (2020, 18 de junio). "Coronavirus: cierran salas alternativas porteñas". En La Nación, Espectáculos (en línea). Consultado el 8 de junio de 2021. Disponible en https://bit.ly/3it3pHE

"Defensoría del Pueblo de la Ciudad de Buenos Aires (2021). Normativa cultura independiente. Medidas y acciones gubernamentales que atañen a trabajadores, organizaciones y espacios de la cultura independiente de la CABA, en el marco de la pandemia del COVID-19

»Estravis Barcala, J. C. (2014). El entre-nos de la cultura. Condiciones estructurales y producciones simbólicas en la escena cultural independiente de la ciudad de Buenos Aires (2008-2013), Tesis de Maestría [inédita].

» Fernández, C. I. (2020). “Estado y políticas culturales en Argentina. Un análisis comparativo entre el Kirchnerismo y la Alianza Cambiemos (2007-2017)". Sociohistórica, Año 45 (102), (en línea). Consultado el 2 de marzo de 2021. Consultado el 7 de junio de 2021. Disponible en https://bit.ly/3x7bPZb

"Fuentes Firmani, E. (2020). “Operación rescate. La cultura argentina frente a la pandemia”. Buenos Aires: RGC Ediciones (en línea). Consultado el 8 de junio de 2021. Disponible en https://bit.ly/2Texyj2

» García Canclini, N. (1987). Políticas culturales en América Latina. Buenos Aires: Editorial Grijalbo.

» Grimson, A. (2014). "Introducción. Políticas para la justicia cultural” en Grimson, A. (comp.), Culturas políticas y políticas culturales (pp. 9 - 14), Buenos Aires: Fundación de Altos Estudios Sociales.

» Mauro, K. (2018). "Entre el mundo del arte y el mundo del trabajo. Herramientas conceptuales para comprender la dimensión laboral del trabajo artístico". telondefondo Revista de Teoría y Crítica teatral. Año XIV, № 27. (en línea). Consultado el 20 de abril de 2021. Disponible en https://bit.ly/3jcTVjs .

» Mauro, K. (2020). “Tenemos que hablar [de números], Torvaldo". Buenos Aires: RGC Ediciones (en línea). Consultado el 8 de junio de 2021. Disponible en https://bit.ly/3crS1Yq

" Ministerio de Cultura de la Nación (2020). Programas e incentivos de asistencia a los sectores culturales en el marco del COVID-19. 
» Observatorio Universitario de Buenos Aires (2020). Cultura en tiempos de pandemia. Publicado el 13 de junio del 2020. Disponible en https://bit.ly/3DezzpT

"Palacios, R. (2020). “El estado de sitio. Las artes escénicas y el COVID-19”. Buenos Aires: RGC Ediciones (en línea). Consultado el 8 de junio de 2021. Disponible en https://bit.ly/2TPRQ2 W

"Rozenholc, A. (2015). Análisis de los subsidios públicos otorgados a las cooperativas de teatro y a las salas o espacios teatrales pertenecientes al circuito de producción alternativo de la Ciudad Autónoma de Buenos Aires. Los casos del Fondo Nacional de las Artes, el Instituto Proteatro y el Instituto Nacional del Teatro durante el período 2000-2010, Tesis de Maestría [inédita].

"Sabatés, P. (2020, 21 de agosto). "Los trabajadores de la cultura declararon la emergencia en CABA”. En Página 12, Cultura y espectáculos (en línea). Consultado el 8 de junio de 2021. Disponible en https://bit.ly/3pAAogg

》Schraier, G. (2008). Laboratorio de producción teatral I. Buenos Aires: Atuel.

»Yaccar, M. D. (2020, 17 de Marzo). “Actores y actrices en emergencia por el coronavirus”. En Página 12, Cultura y espectáculos (en línea). Consultado el 8 de junio de 2021. Disponible en https://bit.ly/3couYxF

»Yaccar, M. D. (2020, 9 de mayo). “Coronavirus: la crisis del teatro independiente”. En Página 12, Cultura y espectáculos (en línea). Consultado el 8 de junio de 2021. Disponible en https://bit.ly/3g97Y8x

"Yaccar, M. D. (2021, 5 de febrero). "El nuevo panorama teatral en la pandemia". En Página 12, Cultura y espectáculos (en línea). Consultado el 8 de junio de 2021. Disponible en https://bit.ly/351OFYk

»Yaccar, M. D. (2021, 20 de marzo). "Entre el pasaje a lo virtual y la precarización”. En Página 12, Especiales P12 (en línea). Consultado el 8 de junio de 2021. Disponible en https://bit.ly/2SnjJyN

»Yaccar, M. D. (2020, 16 de diciembre). "La cultura independiente porteña, “en riesgo”. Página 12, Cultura y espectáculos (en línea). Consultado el 8 de junio de 2021. Disponible en https://bit.ly/3w60QNU

"Yaccar, M. D. (2020, 26 de marzo). "La escena teatral, entre el alivio y la espera”. Página 12, Cultura y espectáculos (en línea). Consultado el 8 de junio de 2021. Disponible en https://bit.ly/3pwMfud

"Yaccar, M. D. (2020, 13 de abril). “Nuevo fondo para espacios culturales”. Página 12, Cultura y espectáculos (en línea). Consultado el 8 de junio de 2021. Disponible en https://bit.ly/zzcsWuw

"Yaccar, M. D. (2020, 18 de junio). "Salas teatrales, entre la pandemia y la burocracia”. Página 12, Cultura y espectáculos (en línea). Consultado el 8 de junio de 2021. Disponible en https://bit.ly/3gewrla

\section{Otros materiales consultados}

» Heger, L. (2021), “Entrevista a Alejandra Carpineti”. En canal de YouTube Estudios interdisciplinarios sobre trabajo y artes (en línea). Consultado el 6 de junio de 2021. Disponible en https://youtu.be/EDEvE_tG4lg 